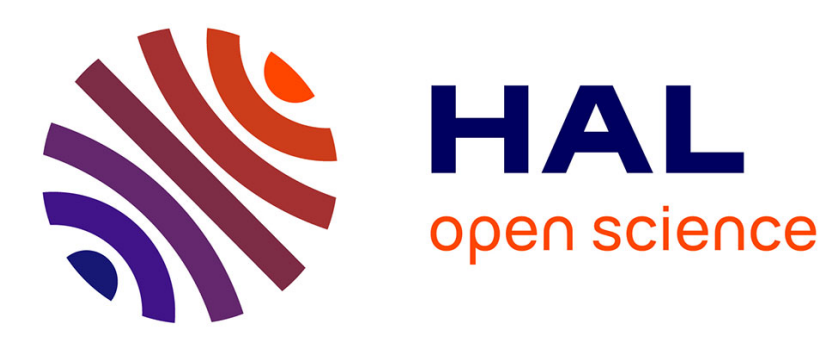

\title{
Une opération à cœur ouvert sous acupuncture? Décryptage d'une émission diffusée sur France 2
}

Nelly Darbois, Jean-Noël Evain, Albin Guillaud, Marc Lilot, Nicolas Pinsault

\section{To cite this version:}

Nelly Darbois, Jean-Noël Evain, Albin Guillaud, Marc Lilot, Nicolas Pinsault. Une opération à cœur ouvert sous acupuncture? Décryptage d'une émission diffusée sur France 2. Santé Publique, 2018, 30 (4), pp.455-464. 10.3917/spub.185.0455 . hal-01917124

\section{HAL Id: hal-01917124 https://hal.science/hal-01917124}

Submitted on 9 Nov 2018

HAL is a multi-disciplinary open access archive for the deposit and dissemination of scientific research documents, whether they are published or not. The documents may come from teaching and research institutions in France or abroad, or from public or private research centers.
L'archive ouverte pluridisciplinaire HAL, est destinée au dépôt et à la diffusion de documents scientifiques de niveau recherche, publiés ou non, émanant des établissements d'enseignement et de recherche français ou étrangers, des laboratoires publics ou privés. 
Une opération à cœur ouvert sous acupuncture ? Décryptage d'une émission diffusée sur France 2.

Open heart surgery under acupuncture? Decrypting a program broadcast on France 2.

L’acupuncture sous les projecteurs.

Acupuncture in prime time.

Auteurs : Nelly Darbois, Jean-Noël Evain, Albin Guillaud, Marc Lilot, Nicolas Pinsault

Revue : Santé Publique

https://www.cairn.info/revue-sante-publique-2018-4-page-455.htm

Version postprint 


\section{Résumé}

Objectifs : Analyser une séquence de l'émission télévisée « Acupuncture, ostéopathie, hypnose : les médecines complémentaires ont-elles de super-pouvoirs? », présentant un exemple d'« une opération à cœur ouvert, avec une anesthésie sous acupuncture » pratiquée dans un hôpital chinois. Proposer une explication plus rationnelle des phénomènes observés. Décrire les processus amenant une chaîne du service public audiovisuel à proposer ce type de séquence à une heure de grande écoute et les conséquences potentielles en matière de santé publique.

Méthode: Analyse critique utilisant des principes et outils rationnels, accompagnés d'une recherche bibliographique sur les bases de données Medline, Google Scholar et Cochrane Library.

Résultats : Les informations délivrées dans la séquence sont ambiguës et ne permettent pas de se faire un avis éclairé sur l'intérêt de l'acupuncture pour une chirurgie à cœur ouvert. Il est raisonnable de penser que la séquence montre une chirurgie pratiquée avec anesthésie péridurale non avouée, couplée à une sédation intraveineuse légère. La particularité est ici la surestimation de l'effet spécifique de l'acupuncture ajoutée au protocole. Les médias ont en effet tendance à exagérer les risques et les effets attendus des traitements sur lesquels ils communiquent, ce qui peut conduire les patients à se tourner vers des thérapies non éprouvées.

Conclusion : La diffusion d'une telle séquence à une heure de grande écoute souligne l'impérieuse nécessité pour le grand public mais aussi et surtout les professionnels de santé d'être formé au tri et à l'analyse critique des informations en matière de santé. Mots clés : pensée critique, acupuncture, anesthésie, mass-medias 


\section{Summary}

Objectives: To analyse a clip from the programme "Acupuncture, osteopathy, hypnosis: do complementary medicines have superpowers?" about acupuncture as an anaesthetic for surgical procedures in China. To propose a rational explanation of the phenomena observed. To describe the processes leading a public service broadcasting channel to offer this type of content at prime time and the potential consequences in terms of public health.

Method: Analysis using critical thinking attitudes and skills and through a bibliographic search of Medline, Google Scholar and Cochrane Library databases.

Results: The information delivered in the television clip is ambiguous. It does not allow the viewer to make an informed opinion on the relevance of acupuncture as an anaesthetic for surgical proceduress. It is reasonable to assume that the clip shows surgery performed with undisclosed epidural anaesthesia coupled with mild intravenous anaesthesia, sometimes performed in other countries. What needs to be highlighted here is the overestimation of acupuncture added to the protocol. The media tend to exaggerate the risks and expected effects of the treatments they report on, which can lead patients to turn to unproven therapies.

Conclusion : The broadcast of such a clip at prime time underlines the urgent need for the public and all health professionals to be trained in the sorting and critical analysis of health information.

Key words: critical thinking, acupuncture, anesthesia, mass media 


\section{Introduction}

Le 7 mars 2017, France 2 a diffusé à 20 h 45 un numéro de son magazine « Les pouvoirs extraordinaires du corps humain » intitulé "Acupuncture, ostéopathie, hypnose : les médecines complémentaires ont-elles de super-pouvoirs?». Le reportage, rediffusé plusieurs fois depuis, a connu une audience importante de l'ordre de 3,7 millions de téléspectateurs le 7 mars 2017 (données de Médiamétrie). Durant une des séquences de l'émission, les deux présentateurs (Adriana Karembeu, AK et Michel Cymes, MC), assistent en Chine à « une opération à cœur ouvert, avec une anesthésie sous acupuncture », qui est présentée comme exemple prouvant « une bonne fois pour toutes l'efficacité de l'acupuncture » par un médecin anesthésiste et acupuncteur, le Dr Cheng $(\operatorname{Dr} C)$.

L'acupuncture permet-elle réellement une opération à cœur ouvert sans autre anesthésie? Cette séquence prouve-t-elle l'efficacité de l'acupuncture en général ? Le téléspectateur reçoit-il suffisamment d'éléments pour se faire un avis rationnel sur ces deux questions?

L'objectif de ce travail est de proposer des réponses rationnelles par le biais d'une analyse critique de la séquence en question, en utilisant des principes et outils formalisés ainsi que les données de la littérature. Les processus de médiatisation qui conduisent à présenter une telle séquence sur une chaîne du service public à une heure de grande écoute, ainsi que ses conséquences potentielles en matière de santé publique, sont également discutés. 


\section{Méthodes}

2.1. Méthode d'extraction des informations de la séquence

Plusieurs démarches ont été effectuées pour travailler sur des informations fiables. Afin de ne pas déformer les propos tenus, l'intégralité des informations orales de l'extrait a été retranscrite de façon littérale et analysée sur le fond et sur la forme. La traduction des passages en chinois a été vérifiée par deux traducteurs sinophones. Pour extraire les informations médicales techniques éventuellement non mentionnées oralement mais présentes sur les images, la séquence a été examinée par deux médecins anesthésistes. Enfin les auteurs ont tenté d'obtenir des informations supplémentaires en contactant directement France Télévisions et l'hôpital Shu Guang à Shanghai, Chine.

\subsection{Outils d'autodéfense intellectuelle}

L'analyse critique proposée se base sur des outils et principes formalisés dans des ouvrages et enseignements d'« esprit critique » («critical thinking »), parfois synonyme d'« autodéfense intellectuelle ». L'esprit critique mêle un ensemble d'aptitudes et de dispositions permettant une analyse, un tri et une évaluation efficaces des informations, de leurs sources, et des arguments invoqués pour affirmer leur véracité (1). Il ne s'agit pas d'une méthode au sens strict du terme. L'« esprit critique » peut être vu comme la formalisation de raisonnements que les individus vont appliquer au quotidien de manière intuitive avec plus ou moins de succès et constance (2). L'art de penser avec ordre et méthode a été formalisé au fil des siècles depuis les philosophes sceptiques de

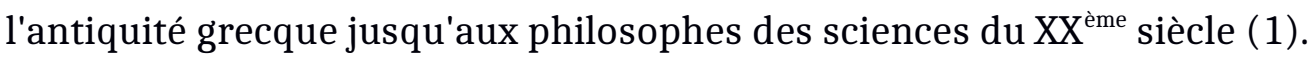

Le tableau I propose une synthèse des outils mobilisés pour cette analyse.

2.2.1. Fécondité de l'alternative et parcimonie des hypothèses 
Face à un phénomène extraordinaire, l'individu sceptique commence par appliquer la maxime «l'alternative est féconde ». Face à un phénomène extraordinaire il faut envisager un ensemble d'explications alternatives. Ensuite, lorsque l'individu dispose de plusieurs hypothèses susceptibles d'expliquer le phénomène en question, il peut appliquer le principe de parcimonie des hypothèses (aussi appelé rasoir d'Occam) (1). Ce principe veut que parmi les hypothèses envisagées, il faille privilégier la plus simple à expliquer (celle qui fait appel aux moins d'entités non démontrées). Cependant si l'hypothèse la plus simple à expliquer doit être privilégiée dans un premier temps, elle ne sera pas forcément l'hypothèse finalement retenue.

\subsubsection{Argument d'autorité et caution scientifique de l'information}

Dans les médias, les faits extraordinaires sont souvent exposés avec des personnalités scientifiques présentées comme des garants. Leurs dires, leurs réactions, ou leur simple présence peuvent influencer le crédit que les spectateurs accordent à l'information (1). Mais en aucun cas ils ne peuvent constituer en eux-mêmes une garantie de la véracité d'une information.

2.2.3. Valeur de l'exemple isolé, appel au témoignage et généralisation abusive Lorsqu'une observation est faite sur un échantillon de population, peut-on raisonnablement faire l'hypothèse qu'elle est valable pour l'ensemble de la population? Le risque de généralisation abusive doit être systématiquement pris en compte. La taille de l'échantillon et son caractère représentatif ou non de la population source font partie des éléments à évaluer. Dans cette optique, la fiabilité d'une observation isolée ou répétée un petit nombre de fois est faible. De même, un témoignage isolé ou un petit nombre de témoignages ne sauraient constituer une preuve d'un phénomène (3).

2.2.4. Tri sélectif des informations et biais de mémorisation 
Lorsqu'un grand nombre d'informations lui parviennent de façon simultanée, l'individu a tendance à les retenir de façon sélective en privilégiant celles lui paraissant étranges, inhabituelles, ou marquantes (4). Cette tendance peut être accentuée par une mise en scène qui présente, volontairement ou non, les informations étranges ou marquantes de manière accentuée. Dans cette situation, il convient de prendre le recul nécessaire, de remonter aux faits objectifs, et d'interpréter avec méfiance ses propres souvenirs et ceux des autres témoins.

2.2.5. Influence des mots utilisés sur la perception d'un événement Le choix des mots pour présenter un fait n'est pas anodin et peut avoir des conséquences propres.

D’abord, les mots peuvent être employés à mauvais escient. Lorsqu'une chose est désignée par un mot qui peut référer à d'autres choses, on parle d'« effet paillasson » en référence à la phrase « essuyez vos pieds » inscrite sur bon nombre de paillassons, dans cet exemple qu'on doit à Henri Broch. Il existe un risque de confusion pouvant conduire à des représentations erronées d'autant plus grandes lorsque l'information présentée utilise un registre scientifique spécifique moins maîtrisé par les spectateurs (1). Ensuite, le fait d'utiliser un mot plutôt qu'un autre peut influencer les représentations. Par exemple, le fait d'intituler une brochure présentant une méthode pédagogique " Right brain " plutôt que « Right start », pour un contenu identique, conduit à ce que les individus trouvent le fondement scientifique de la méthode plus fort (5). Deux mots peuvent avoir un même sens mais des connotations différentes, positive ou négative. On parle de mot à " effet impact " lorsqu'un mot a, en plus de sa signification stricte, une connotation émotionnelle qui aurait été absente s'il avait été substitué par un synonyme plus « neutre» $(1)$ 
Enfin, certains registres lexicaux peuvent également influencer la perception d'un événement. Le registre laudatif, par exemple, très fréquent dans les médias, consiste en l'utilisation d'une abondance de qualificatifs mélioratifs et d'exclamations admiratives tels que «Formidable ! », «C'est exceptionnel! », «Inouï!»(1). L'identification d'un registre appelant les émotions dans une information présentée comme scientifique devrait accentuer la prise de recul chez le spectateur.

\subsection{Recherche bibliographique}

Une recherche bibliographique d'articles scientifiques (en français et en anglais) sur les bases de données Medline ${ }^{1}$, Google Scholar et Cochrane Library et sur un moteur de recherche généraliste a été effectuée. Cette recherche bibliographique avait une visée exploratoire sans prétendre à l'exhaustivité ou à la systématicité. Trois objectifs ont été poursuivis. Premièrement, faire un bref état des lieux de la littérature scientifique existante concernant la chirurgie cardiaque sous acupuncture. Deuxièmement, savoir s'il existe, dans le monde, des chirurgies cardiaques réalisées sans anesthésie générale ni acupuncture. Troisièmement, réaliser une synthèse des preuves existantes de l'efficacité de l'acupuncture comme adjuvant anesthésique.

\footnotetext{
${ }^{1}$ Formule : (acupuncture [tiab] or electroacupuncture [tiab] or electro-acupuncture [tiab]) and (anesthesia [tiab] or anaesthesia [tiab] or anesthesic [tiab] or anaesthesic [tiab]) and (review [tiab] or "meta-analysis" [tiab])
} 


\section{Résultats}

\subsection{Description factuelle de la séquence}

La séquence se déroule dans un bloc opératoire de l'hôpital de Shu Guang, à Shanghai, en Chine. Une patiente d'une trentaine d'année est programmée pour avoir une chirurgie valvulaire avec une "anesthésie sous acupuncture ». Une dizaine de personnes sont présentes en salle d'opération. AK rentre en salle mais $\mathrm{MC}$ reste derrière la porte vitrée. La patiente est installée sur la table d'opération. Elle n'est pas intubée. Elle est équipée de lunettes à oxygène, d'une sonde nasogastrique, d'une voie veineuse centrale, d'un cathéter artériel radial, et d'un capteur électroencéphalographique. Les images du scope montrent qu'elle est monitorée par ECG, Sp02, BIS ${ }^{\circledR}$, pression artérielle invasive, pression veineuse centrale et température. Aucun dispositif de contrôle des voies aériennes n'est présent et la patiente ouvre les yeux de façon intermittente. Une personne lui insère des aiguilles d'acupuncture au niveau du membre supérieur gauche, trente minutes avant l'incision. Les images suivantes montrent l'incision thoracique. À ce moment, la patiente cligne des yeux plusieurs fois, mais ne semble pas avoir mal. Après la sternotomie, qui n'est pas montrée, AK décrit qu'elle voit le cœur battre. Puis une sédation profonde est administrée par voir intra-veineuse, avant la mise en place d'une circulation extracorporelle et l'arrêt transitoire du cœur de la patiente. Après la réparation de la valve, les battements cardiaques reprennent. AK rejoint alors $\mathrm{MC}$ et $\mathrm{Dr} \mathrm{C}$ en dehors de la salle d'opération. À la fin de la séquence, le $\operatorname{Dr} C$ indique que l'acupuncture a permis de réduire de deux tiers la dose de " tranquillisants " pour cette intervention, et que « dans certains cas on peut remplacer toute l'anesthésie générale par l'anesthésie par acupuncture ». 
Les deux sinophones ayant retraduit indépendamment les deux passages en chinois n'ont pas relevé d'erreurs dans la traduction proposée dans l'émission, bien que la superposition des voix rend cette vérification difficile. D’autre part, les services clients de France télévisions ont répondu qu'il n'était pas possible de communiquer des informations personnalisées autres que celles diffusées à l'antenne. Enfin, malgré de multiples appels à l'hôpital de Shu Guang depuis la Chine, une mise en relation avec l'équipe ayant participé au tournage fut impossible.

\subsection{Analyse critique}

Le tableau II illustre les outils présentés dans la partie méthode, à partir de l'analyse de l'extrait.

Avant toute chose, il faut souligner qu'un seul exemple, renforcé par des témoignages, est retenu ici comme une preuve de l'efficacité de l'acupuncture pour la chirurgie cardiaque, et de l'efficacité de l'acupuncture en général : «je crois que je sais comment vous prouver une bonne fois pour toute l'efficacité de l'acupuncture » ou plus loin « Moi l'acupuncture c'est vrai que j'avais un peu du mal, j'avais une idée sur l'acupuncture mais là je suis devant des faits (...) ». Il s'agit ici d'une généralisation abusive qui sous-entend une conclusion générale quant à l'efficacité de l'acupuncture, toute pathologie ou symptôme confondus, à partir d'un cas unique.

D’autre part, $\mathrm{MC}$, grâce à son statut de médecin, est posté comme référent scientifique de cette émission, et sa popularité auprès du grand public (présentateur du Magazine de la santé) renforce cet argument d'autorité.

L'anesthésiologie est une spécialité médicale mal connue du grand public mais aussi du reste de la communauté médicale. Les termes techniques sont spécifiques et le risque de confusion élevé. Dans cette séquence, ce risque est renforcé par un effet paillasson : le 
terme " anesthésie » est ici employé pour désigner deux choses différentes qui sont l'anesthésie et la sédation. Dans sons sens strict, l'anesthésie désigne la suppression totale de toute sensibilité. Elle peut être de type générale (associée à une perte de conscience totale) ou locorégionale (concernant seulement une partie du corps). La sédation est une diminution du niveau de conscience induite par des médicaments. Elle augmente le confort du patient mais ne permet pas de supprimer pas la sensibilité douloureuse. Les médicaments utilisés pour générer une sédation peuvent être les mêmes que ceux d'une anesthésie générale, mais les doses sont plus élevées pour cette dernière. De plus, l'expression « anesthésie sous acupuncture » est source de confusion. Désigne-t-elle une anesthésie prodiguée par de l'acupuncture, une anesthésie complétée par de l'acupuncture, ou encore une anesthésie remplacée par de l'acupuncture ? Dr C ne clarifie pas cette ambiguité. Les notions de "tranquillisants " et d'" anesthésie ", pourtant très distinctes, sont confondues. Des expressions à « effet impact » favorables à l'acupuncture (exemple : "quelques tranquillisants ») sont privilégiées. Le choix est fait par les journalistes de parler majoritairement de l'acupuncture (" acupuncture » est prononcé 12 fois dans la séquence et « aiguilles » 3 fois) plutôt que des médicaments anesthésiques reçus par la patiente (« sédatifs » et « tranquillisants » ne sont prononcés que 2 fois chacun). Les téléspectateurs retiennent une information tronquée et qui incite à la surestimation de l'effet spécifique de l'acupuncture durant cette chirurgie.

Pendant toute la séquence, la voix hors champ et les présentateurs insistent sur les émotions des témoins de la scène. L'accent est mis sur le caractère extraordinaire, avec l'utilisation d'un registre laudatif abondant avec comme paroxysme l'émotion d'AK lorsqu'elle voit un cœur battre pour la première fois. On retrouve de façon omniprésente des expressions comme "jamais vu ", "dépasse tout entendement ", " incroyable », « extraordinaire », « bouche bée », ou encore « magie ». La musique de fond choisie pour 
le montage renforce cette accentuation. L'expression « tout le monde retient son souffle » au moment de la CEC apporte un aspect sensationnel supplémentaire, non justifié par le risque anesthésique ou chirurgical, et sans lien avec l'acupuncture. Très peu de temps est laissé aux explications rationnelles, de façon à ce que le téléspectateur mémorise de façon préférentielle le caractère extraordinaire en lui-même.

Tous ces éléments montrent que cette séquence peut, malgré une très faible valeur argumentative, persuader le téléspectateur de l'authenticité de la situation décrite. Cependant, ils ne fournissent pas en eux-mêmes d'explication rationnelle à la situation présentée.

3.3 Résultats de recherches bibliographiques

\subsubsection{Chirurgie cardiaque sous acupuncture}

La « chirurgie cardiaque sous acupuncture » en Chine est décrite depuis 1974 dans les bases de données consultées (6,7). À l'époque, la promotion de l'acupuncture dans ce contexte chirurgical se faisait déjà via des timbres postaux (8). En 2011, « l'anesthésie par acupuncture pour chirurgie à cœur ouvert en Chine contemporaine » a fait l'objet d'une publication dans International Journal of Cardiology (9) par une équipe de l'hôpital Shu Guang en Chine. Le délai de un jour entre la soumission (13/04/2011) et l'acceptation (14/04/2011) de l'article, paraît incompatible avec une relecture par les pairs. L'acupuncture est présentée comme un moyen d'éviter l'anesthésie générale, l’intubation et la morbidité qui lui sont associées, et donc de réduire les dépenses liées à la chirurgie cardiaque. Une comparaison rétrospective est faite entre 100 patients opérés sous anesthésie générale (AG) et 100 patients opérés sous « acupuncture combinée à une anesthésie médicale ». Le protocole utilisant de l'acupuncture prévoit avant l'incision l'administration de $1 \mathrm{mg} / \mathrm{kg}$ de morphine intramusculaire, de $0.1 \mathrm{mg}$ 
fentanyl intraveineux (IV) et de $2 \mathrm{mg}$ de midazolam IV, avec possibilité de « répéter en cas de besoin » les doses de fentanyl et de midazolam. De plus, il prévoit une injection sous-cutanée d'anesthésique local (20 à $30 \mathrm{~mL}$ de lidocaïne $2.5 \mathrm{mg} / \mathrm{mL}$ ). Dans les résultats comparant les deux groupes, les doses totales de fentanyl sont comme attendu plus élevées dans le groupe AG. Cependant les doses totales de morphine, midazolam et lidocaïne ne sont pas présentées. Enfin, l'intérêt d'une éventuelle anesthésie péridurale n'est pas discuté dans l'article.

Or, la littérature indique que l'anesthésie péridurale est largement utilisée en Chine depuis les années 1950 (10), que les techniques d'anesthésie régionale sont tout à fait compatibles avec l'acupuncture $(11,12)$, et que la péridurale est largement utilisable en chirurgie cardiaque (13). Les publications relatives à la " chirurgie cardiaque sous acupuncture " mentionnent souvent une anesthésie locale et une sédation en complément de l'acupuncture mais ne discutent pas les techniques d'anesthésie régionales qui peuvent être associées. Un parallèle peut être fait avec l'appendicectomie réalisée en urgence sur James Reston, un journaliste du New York Times accompagnant Richard Nixon en Chine en 1971. Si des publications à ce sujet relatent " une appendicectomie sous acupuncture ", James Reston a révélé lui-même dans les suites qu'une injection d'anesthésique local a été pratiquée dans son dos au préalable $(14,15)$.

\subsubsection{Chirurgie cardiaque sans anesthésie générale}

La chirurgie cardiaque sur patient éveillé est réalisée aussi ailleurs sans acupuncture. Des pontages coronaires sur cœur battant ont d'abord été décrits (16-19), mais aussi des chirurgies de remplacement valvulaires $(20,21)$ avec mise en place d'une CEC. La technique de chirurgie cardiaque sur patient éveillé a été décrite avec précision (22). La douleur de la chirurgie est supprimée avec une anesthésie péridurale thoracique haute. 
Le patient peut ressentir un inconfort ou une anxiété nécessitant de lui administrer une sédation intraveineuse, induisant un sommeil réversible avec maintien de la ventilation spontanée (VS). La phase de CEC nécessite souvent d'augmenter les doses de médicaments, avec la possibilité que le patient arrête de respirer. Cependant, dans cette situation très précise, le recours à une assistance respiratoire n'est pas nécessaire puisque la CEC assure en parallèle l'oxygénation du sang (22).

\subsubsection{Acupuncture comme adjuvant anesthésique}

Quatre revues systématiques ou méta-analyses sont, à notre connaissance, publiées sur ce sujet.

La première porte sur l'efficacité de l'acupuncture comme adjuvant analgésique d'une anesthésie standard. Elle conclut, après analyse de 19 essais contrôlés randomisés, à un niveau de preuve insuffisant sur l'efficacité de l'acupuncture versus absence d'acupuncture (23).

Une seconde porte sur l'efficacité de l'électroacupuncture comme méthode de sédation consciente pour soulager la douleur d'une ponction ovocytaire transvaginale dans un contexte de procréation médicalement assistée. Elle conclut à une absence de supériorité démontrée de cette méthode par rapport aux méthodes antalgiques conventionnelles (23), ce qui est confirmé par une revue de 2013 (25).

La troisième est une méta-analyse sur l'efficacité de l'electroacupuncture pour 8 critères de jugement per et post-opératoires en complément d'une anesthésie générale versus anesthésie générale seule, dans un contexte de chirurgie cardiaque (26). Les auteurs concluent favorablement pour 4 critères. Cependant, les études incluses sont en faible nombre (de 2 à 4 en fonction des critères de jugement), mobilisent des échantillons 
réduits (20 à 70 patients) et présentent pour la plupart des incertitudes sur leur risque de biais ou des risques de biais élevés.

Enfin, une méta-analyse porte sur l'efficacité de l'électroacupuncture pour divers paramètres per et post-opératoires en complément d'une anesthésie générale versus anesthésie générale seule, dans un contexte de craniotomie (27). Les auteurs suggèrent que l'acupuncture couplée à l'anesthésie générale « a des effets analgésiques supplémentaires, réduit la quantité nécessaire d'anesthésiques volatils, réduit l'apparition de nausées et de vomissements postopératoires et pourrait avoir des effets protecteurs sur les tissus cérébraux ». Cependant 9 des 10 études inclues sont à risque de biais élevé ou incertain concernant l'aveuglement pour l'évaluation des critères de jugements et 6 concernant l'allocation cachée et la procédure d'aveuglement du personnel et des participants.

La littérature ne permet pas à ce jour d'affirmer une efficacité spécifique de l'acupuncture comme adjuvant anesthésique dans les contextes chirurgicaux explorés.

\subsection{Hypothèses explicatives}

\subsubsection{Hypothèse retenue par le reportage}

Dans le reportage, l'acupuncture est présentée comme permettant de remplacer partiellement ou totalement l'anesthésie générale durant la chirurgie cardiaque, avec un effet analgésique propre considéré comme « extraordinaire » et permettant à la patiente de « trouver en elle-même le moyen de court-circuiter la douleur ». 


\subsubsection{Propositions d’hypothèses alternatives}

L'analyse critique du reportage et les données issues des recherches bibliographiques permettent d'appliquer ici le principe de fécondité de l'alternative en émettant deux hypothèses alternatives rationnelles plus probables que celle avancée dans le reportage. Première hypothèse alternative : les doses de médicaments anesthésiques intraveineux et locaux utilisés en parallèle de l'acupuncture seraient plus élevées que le mot «tranquillisants » le laisse entendre. La patiente serait endormie par les anesthésiques IV de courte durée d'action administrés en continu. Selon le dosage, modifiable en temps réel, le niveau de conscience de la patiente varierait entre celui d'une sédation, avec des éveils intermittents, et celui d'une anesthésie générale, notamment pendant la phase de CEC. Cependant, pour procurer une analgésie permettant la sternotomie, il faudrait des doses de médicaments intraveineuse incompatibles avec le maintien d'une VS, et une assistance ventilatoire serait nécessaire. Or aucune assistance ventilatoire n'est observable dans le reportage.

Seconde hypothèse alternative : en complément du protocole décrit, une anesthésie péridurale (permettant de supprimer tous les stimuli sensitifs, y compris la douleur) serait réalisée par l'équipe anesthésique, mais non mentionnée dans le reportage. Les plans sur la main gauche de la patiente montrent qu'elle est totalement immobile en position neutre. Cette immobilité « non naturelle » chez une patiente éveillée suggère une paralysie de ce membre, facilement explicable par le bloc moteur induit par l'anesthésie péridurale.

\subsubsection{Hypothèse alternative retenue}

L'application du principe de parcimonie des hypothèses fait privilégier la plus simple à expliquer pour rationaliser la situation présentée. Il s'agit de la présence dissimulée 
d'une anesthésie péridurale pour interrompre les stimuli sensitifs induit par la chirurgie, en complément du protocole décrit. Cette anesthésie péridurale a pu être réalisée en injection unique dorsale avant l'installation chirurgicale ou bien avec la mise en place d'un cathéter pour entretenir l'anesthésie péridurale durant la chirurgie. Une sédation IV, permettant un maintien de la VS (sauf pendant la CEC) et des éveils intermittents est compatible avec l'hypothèse d'une péridurale.

L'information donnée par le $\mathrm{Dr} \mathrm{C}$ à la fin de la séquence est compatible avec cette hypothèse si elle est comprise sous l'angle suivant : la chirurgie était auparavant réalisée avec une anesthésie péridurale complétée par sédation IV, comme décrit dans la littérature (paragraphe 3.3.2), et l'acupuncture aurait permis, depuis son ajout au protocole anesthésique, de réduire les doses de médicaments nécessaires pour la sédation. 


\section{Discussion}

L'analyse critique et les recherches bibliographiques ont conduit à énoncer d'autres hypothèses explicatives que l'efficacité spécifique de l'acupuncture lors de l'intervention chirurgicale présentée dans la séquence du reportage. Il semble raisonnable de favoriser l'hypothèse d'une intervention pratiquée avec une anesthésie péridurale, complétée par une sédation IV.

Ce n'est pas la première fois qu'un reportage sur l'acupuncture suscite le débat. En 2006 la British Broadcasting Corporation (BBC), au Royaume-Uni, a diffusé dans une émission intitulée «Alternative Medicine, The Evidence » un reportage similaire consacré à une opération chirurgicale à cœur ouvert avec acupuncture. Plusieurs universitaires, médecins et citoyens ont dénoncé la façon dont la $\mathrm{BBC}$ avait présenté cette intervention (28). En 1998, Isidore Rosenfeld, un cardiologue états-unien, rapportait dans un magazine avoir observé dans les années 70 en Chine une opération chirurgicale à cœur ouvert avec de l'acupuncture en guise de procédure anesthésique. Ce témoignage avait été critiqué par un spécialiste en médecine interne (14). Enfin dans les années 70, d'autres articles de presse grand public et médicale (dont le Journal of the American Medical Association) relayèrent des informations sur l'utilisation de l'acupuncture lors de chirurgies en Chine, et furent par la suite dénoncés pour avoir présenté les faits de manière erronée (14).

Ces différentes affaires illustrent à quel point il est facile, concernant la santé, de trouver, dans les médias grand public comme scientifiques, un traitement de l'information détourné ou scénarisé. La simplification et la stimulation de l'émotion des destinataires sont favorisées plutôt que la rationalisation et l'objectivité par la diffusion de toutes les informations nécessaires à la bonne compréhension du phénomène. Cependant, le rasoir 
de Hanlon (voir Tableaux II et II) invite à « Ne jamais attribuer à la malveillance ce que l'ignorance suffit à expliquer. $»^{2}$ (29). Dans cet article, point d'intentions malveillantes ou manipulatrices sont prêtées aux personnes impliquées dans la réalisation et la diffusion de ce reportage. En revanche, la façon de présenter et trier les informations, caractéristique du traitement médiatique réservé aux connaissances scientifiques et "pseudo-scientifiques » notamment dans le champ de la santé, doit être soulignée.

Certains chercheurs font le constat que les médias, les journaux médicaux, les industries des produits de santé ou encore les institutions académiques ont tendance à exagérer les risques et les effets attendus des traitements sur lesquels ils communiquent. Ce phénomène conduit à biaiser les appréciations des patients sur les risques encourus et les espérances sur les traitements proposés (30). Les professionnels de santé peuvent également être influencés par ce procédé médiatique. Une étude récente menée auprès des médecins généralistes français met en évidence un risque plus élevé d'être sensible aux controverses sur les vaccins lorsqu'ils pratiquent occasionnellement des médecines alternatives et qu'ils considèrent les informations fournies par les médias comme fiables (31).

En 2011, le Conseil national de l'ordre des médecins saluait l'arrivée de la lecture critique d'article dans le programme de l'examen classant national depuis 2009 (32), considérant l'« esprit critique » comme "une qualité indispensable au médecin ». De telles formations ne devraient cependant pas se substituer à des enseignements de démarche critique plus large que la seule analyse critique de la littérature scientifique.

\footnotetext{
${ }^{2}$ Dans sa version originale, la phrase est « Never attribute to malice that which is adequately explained by stupidity». Nous avons choisi de remplacer le terme « stupidity " par « ignorance » qui nous semble plus doux et plus approprié.
} 
Récemment, plusieurs professeurs d'université de médecine ont communiqué dans la littérature internationale l'importance de tels enseignements (33,34). Dans le secteur de la santé, l'analyse de thérapeutiques controversées, de l'acupuncture à l'urinothérapie, est un levier stimulant pour implémenter des bases en épistémologie nécessaires à l'élaboration de réflexes d'autodéfense intellectuelle efficaces.

De tels enseignements sont encore rares et manquent dans les programmes officiels (34), notamment en France. L'université Grenoble-Alpes propose depuis 2017 une unité d'enseignement «Santé et autodéfense intellectuelle » accessible aux étudiants des filières médicales et paramédicales (35). Le développement de ces enseignements devrait se faire en parallèle d'études visant à évaluer leur efficacité. En effet, encore peu de données de bonne qualité sont disponibles sur le sujet (36).

Ce travail a permis de présenter certains outils permettant d'améliorer l'analyse critique d'informations en santé. Il a également appliqué ces outils à un contenu qui a été vu par plusieurs millions de téléspectateurs. Des recherches bibliographiques ont été faites pour compléter les informations transmises dans la séquence du reportage. Cependant, ces recherches sont succinctes et nécessiteraient d'être conduites de manière plus systématique et approfondie. D’autre part, des informations sont manquantes pour valider de manière plus certaine l'hypothèse alternative proposée.

Enfin, l'analyse présentée ici est à remettre en perspective avec l'affaire « FakeMed » qui a été fortement médiatisée en mars 2018. Un collectif de 124 professionnels de santé parmi lesquels des médecins de toute spécialité ont rédigé une tribune enjoignant l'Ordre des médecins et les pouvoirs publics d'adopter des mesures pour empêcher l'usage par des professionnels de santé de thérapies non éprouvées telles que l'acupuncture, la mésothérapie et l'homéopathie (37). L'Ordre des médecins en juin 2018 
a dans les suites effectué une " mise au point " sur le cadre déontologique s'imposant à tous les médecins (38).

La séquence sur l'acupuncture et l'anesthésie du reportage de France 2 consacrée aux "pouvoirs extraordinaires du corps humain » délivre des informations incomplètes et ambiguës. Elle ne permet pas de se faire un avis rationnel sur la pertinence du recours à l'acupuncture en tant que technique anesthésique pour une intervention chirurgicale. Cet exemple d'analyse se veut illustratif de la nécessité pour le grand public mais aussi pour les professionnels de santé d'être formé au tri et à l'analyse critique des informations en matière de santé.

\section{Remerciements}

Les auteurs remercient Hugo Muselli, Li Yaho et Richard Monvoisin pour leur aide pour la traduction et les recherches d'informations ainsi que Julien Peccoud et François Lecomte pour leurs relectures. 
Tableau I

Description et illustration des outils utilisés pour l'analyse de l'extrait de l'émission.

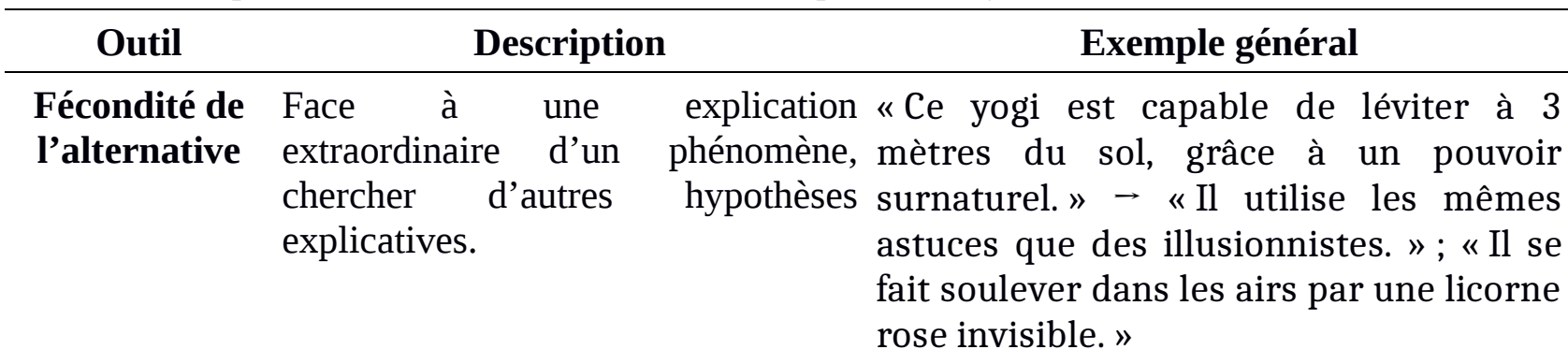

Parcimonie Lorsque plusieurs hypothèses "Il utilise les mêmes astuces que des des hypothèses sont en compétition, privilégier illusionnistes. » d'abord la plus simple à expliquer.

Argument Invoquer une personnalité faisant «L'armement nucléaire est une nuisance, d'autorité autorité dans le domaine concerné le prix Nobel Georges Charpak l'a affirmé n'est pas un gage suffisant de haut et fort. » rigueur.

Appel au Se méfier des expériences « Ma fille à la naissance avait le crâne témoignage personnelles invoquées en guise de tout plat; heureusement, elle a vu un preuve. rebouteux et tout est rentré dans l'ordre. Tu devrais voir un rebouteux!»

Généralisation Éviter de tirer d'un échantillon trop «Je reviens d'un week-end en Corse où abusive petit une conclusion générale. j'ai discuté avec des gens du village; franchement, les Corses, ils ne sont vraiment pas accueillants!»

Tri sélectif des Notre mémoire est faillible, «Les femmes que j’ai croisé sur ma route informations remonter aux faits de la manière la jusqu'à présent roulaient plus fiable possible. systématiquement mal. »

Effet Vérifier si le mot utilisé ne réfère "Essuyez vos pieds" [on essuie ses paillasson pas à autre chose que la chose semelles sur un paillasson et non ses discutée. pieds]

Effet impact Ne pas sous-estimer l'effet d'un mot « Il y a eu des pertes collatérales au Viêt qui aurait pu être substitué par un Nam. » $\rightarrow$ " Des civils sont morts au Viêt autre dont l'effet émotionnel induit Nam.» aurait été différent.

\footnotetext{
Rasoir de Ne jamais attribuer à la malveillance "Ce magnétiseur est un vrai charlatan. » Hanlon ce que l’ignorance suffit à expliquer.
} 
Tableau II

Illustration des outils dans le contexte de l'extrait de l'émission.

\begin{tabular}{|c|c|}
\hline Outil & Exemple du reportage \\
\hline Fécondité de l’alternative & $\begin{array}{l}\text { "Grâce uniquement à des aiguilles, on peut anesthésier une } \\
\text { personne et l'opérer à cœur ouvert. " } \rightarrow \text { "Cette personne } \\
\text { recevait également des médicaments anesthésiques } \\
\text { intraveineux. "; "Cette personne bénéficiait en parallèle } \\
\text { d'une anesthésie régionale." }\end{array}$ \\
\hline Parcimonie des hypothèses & $\begin{array}{l}\text { «Cette personne bénéficiait en parallèle d'une anesthésie } \\
\text { régionale. » }\end{array}$ \\
\hline Argument d'autorité & $\begin{array}{l}\text { Présence de médecins dont au moins un est une personnalité } \\
\text { médiatique. }\end{array}$ \\
\hline Appel au témoignage & $\begin{array}{l}\text { «J'ai vu une patiente se faire opérer à cœur ouvert } \\
\text { uniquement sous acupuncture, l'acupuncture, ça marche } \\
\text { vraiment!" }\end{array}$ \\
\hline Généralisation abusive & $\begin{array}{l}\text { "Si l'acupuncture est efficace pour l'anesthésie en chirurgie } \\
\text { cardiaque sur cette patiente, alors c'est une technique } \\
\text { vraiment efficace." }\end{array}$ \\
\hline Tri sélectif des informations & $\begin{array}{l}\text { "J'ai vu une patiente se faire opérer à cœur ouvert, sans } \\
\text { médicaments, uniquement grâce à l'acupuncture. " }\end{array}$ \\
\hline Effet paillasson & $\begin{array}{l}\text { «L'anesthésie se réduira à quelques sédatifs et à des aiguilles } \\
\text { d'acupuncture. » [la sédation] }\end{array}$ \\
\hline Effet impact & $\begin{array}{l}\text { "La patiente n'a que quelques tranquillisants. " } \rightarrow \text { "La } \\
\text { patiente a quelques médicaments sédatifs. " }\end{array}$ \\
\hline Rasoir de Hanlon & «Ces journalistes, tous des manipulateurs!» \\
\hline
\end{tabular}




\section{Références}

1. Monvoisin R. Pour une didactique de l'esprit critique-Zététique et utilisation des interstices pseudoscientifiques dans les médias. Thèse de didactique des sciences. Université Joseph-Fourier-Grenoble I ; 2007.

2. Baillargeon N. Petit cours d'autodéfense intellectuelle. Lux; 2006.

3. Pinsault N, Monvoisin R. Tout ce que vous n'avez jamais voulu savoir sur les thérapies manuelles. Saint-Martin-d'Hères (Isère): Presses universitaires de Grenoble; 2014. 310p.

4. Nicolas S, Gounden Y. L’imagerie bizarre et la mémoire. Psychologie française. 2011;56(4):203-208.

5. Lindell AK, Kidd E. Consumers Favor "Right Brain" Training: The Dangerous Lure of Neuromarketing. Mind, Brain, and Education. 1 mars 2013;7(1):35-9.

6. Bonica JJ. Acupuncture anesthesia in the People's Republic of China Implications for American medicine. Jama 1974; 229(10): 1317-25.

7. Katz AM. Acupuncture anesthesia for open heart surgery: a case report. The American journal of cardiology. 1974;34(2):250-253.

8. Nguyen J. Anesthésie par acupuncture en chirurgie cadiaque [Internet]. [Cité le 5 avril 2018]. Disponible sur: http://www.meridiens.org/acuMoxi/sixquatre/JOHANCHIRURGIE.htm

9. Zhou J, Chi H, Cheng TO, Chen T, Wu Y, Zhou W, et al. Acupuncture anesthesia for open heart surgery in contemporary China. International journal of cardiology. $2011 ; 150(1): 12-16$.

10. Bonica JJ. Anesthesiology in the People's Republic of China. Anesthesiology: The Journal of the American Society of Anesthesiologists. 1974;40(2):175-186. 
11. Sun P. [A study of subtotal gastrectomy under the acupuncture combined with epidural anesthesia of small dosage]. Zhen Ci Yan Jiu Acupunct Res. 1996;21(2):11-5.

12. Wu G. Acupuncture anesthesia in China: retrospect and prospect. Chinese journal of integrative medicine. 2007;13(3):163-165.

13. Svircevic V, van Dijk D, Nierich AP, Passier MP, Kalkman CJ, van der Heijden GJ, et al. Meta-analysis of thoracic epidural anesthesia versus general anesthesia for cardiac surgery. Anesthesiology: The Journal of the American Society of Anesthesiologists. $2011 ; 114(2): 271-282$.

14. Atwood K. "Acupuncture Anesthesia": A Proclamation from Chairman Mao (Part I) [Internet]. Science-Based Medicine. 2009 [Cité le 21 janvier 2018]. Disponible sur https://sciencebasedmedicine.org/acupuncture-anesthesia-a-proclamation-ofchairman-mao-part-i/

15. Reston J. Now, about my operation in Peking. New York Times. 1971;1(6).

16. Vanek T, Straka Z, Brucek P, Widimsky P. Thoracic epidural anesthesia for offpump coronary artery bypass without intubation. European journal of cardio-thoracic surgery. 2001;20(4):858-860.

17. Aybek T, Kessler P, Dogan S, Neidhart G, Khan MF, Wimmer-Greinecker G, et al. Awake coronary artery bypass grafting: utopia or reality? Ann Thorac Surg. 2003;75(4):1165-70

18. Karagoz HY, Kurtoglu M, Bakkaloglu B, Sonmez B, Cetintas T, Bayazit K. Coronary artery bypass grafting in the awake patient: three years' experience in 137 patients. The Journal of thoracic and cardiovascular surgery. 2003;125(6):1401-1404.

19. Chakravarthy M, Jawali V, Patil TA, Jayaprakash K, Shivananda NV. High thoracic epidural anesthesia as the sole anesthetic for performing multiple grafts in off-pump 
coronary artery bypass surgery. Journal of cardiothoracic and vascular anesthesia. 2003;17(2):160-164.

20. Schachner T, Bonatti J, Balogh D, Margreiter J, Mair P, Laufer G, et al. Aortic valve replacement in the conscious patient under regional anesthesia without endotracheal intubation. The Journal of thoracic and cardiovascular surgery. 2003;125(6):1526-1527. 21. Chakravarthy M, Jawali V, Patil TA, Jayaprakash K, Kolar S, Joseph G, et al. Conscious cardiac surgery with cardiopulmonary bypass using thoracic epidural anesthesia without endotracheal general anesthesia. J Cardiothorac Vasc Anesth. 2005;19(3):300-5.

22. Chakravarthy M. Technique of awake cardiac surgery. Techniques in regional anesthesia \& pain management. 2008;12(1):87-98.

23. Lee H, Ernst E. Acupuncture analgesia during surgery: a systematic review. Pain. $2005 ; 114(3): 511-517$.

24. Stener-Victorin E. The pain-relieving effect of electro-acupuncture and conventional medical analgesic methods during oocyte retrieval: a systematic review of randomized controlled trials. Human reproduction. 2005;20(2):339-349.

25. Kwan I, Bhattacharya S, Knox F, McNeil A. Pain relief for women undergoing oocyte retrieval for assisted reproduction. The Cochrane Library. 2013;

26. Asmussen S, Przkora R, Maybauer DM, Fraser JF, Sanfilippo F, Jennings K, et al. Meta-Analysis of Electroacupuncture in Cardiac Anesthesia and Intensive Care. J Intensive Care Med. 1 juin 2017;0885066617708558.

27. Asmussen S, Maybauer DM, Chen JD, Fraser JF, Toon MH, Przkora R, et al. Effects of acupuncture in anesthesia for craniotomy: a meta-analysis. Journal of neurosurgical anesthesiology. 2017;29(3):219-227. 
28. Singh S. Did we really witness the " amazing power » of acupuncture? Février 2006 [Cité le 20 janvier 2018]. Disponible sur http://www.telegraph.co.uk/news/science/science-news/3344833/Did-we-reallywitness-the-amazing-power-of-acupuncture.html

29. Bloch A. Murphy's Law Book Two. More Reasons Why Things Go Wrong. Price/Stern/Sloan Publishers Inc. New-York; 1980. 95p.

30. Woloshin S, Schwartz LM, Welch HG. Know Your Chances. University of California Press ; 2008. 158p.

31. Le Marechal M, Fressard L, Agrinier N, Verger P, Pulcini C. General practitioners' perceptions of vaccination controversies: a French nationwide cross-sectional study. Clinical Microbiology and Infection. 3 Novembre 2017.

32. Cerfon J. Conflits d'intérêts dans l'exercice médical : La déontologie médicale peut-elle aider à restaurer la confiance? Les conflits d'intérêts : un sujet d'actualité. Conseil national de l'ordre des médecins; 2011. Disponible sur https://www.conseilnational.medecin.fr/article/conflits-d-interets-dans-l-exercice-medical-1086

33. Achilleos H. Beliefs, critical thinking and evidence-based medicine. BMJ Evid Based Med. 2018;23(1):4-5.

34. Sharples JM, Oxman AD, Mahtani KR, Chalmers I, Oliver S, Collins K, et al. Critical thinking in healthcare and education. BMJ. 16 2017;357:j2234.

35. Collectif de Recherche Transdisciplinaire Esprit Critique \& Sciences. Février 2017 : nouveau cours Santé et autodéfense intellectuelle à Grenoble. [Internet]. [Cité le 6 avril 2018]. Disponible sur https://cortecs.org/activites/ca-vient-de-sortir-nouveaucours-sante-et-autodefense-intellectuelle-a-grenoble/ 
36. Lee DS, Abdullah KL, Subramanian P, Bachmann RT, Ong SL. An integrated review of the correlation between critical thinking ability and clinical decision-making in nursing. J Clin Nurs. déc 2017;26(23-24):4065-79.

37. Fake médecine. Tribune [Internet]. Mars 2018. [Cité le 5 avril 2018]. Disponible sur http://fakemedecine.blogspot.com/

38. Conseil National de l'Ordre des Médecins. «Médecines alternatives et complémentaires » [Internet]. Juin 2018 [cité 23 juin 2018]. Disponible sur: https://www.conseil-national.medecin.fr/node/2836 